\title{
PROPIEDADES HIDROGEOQUÍMICAS E ISOTÓPICAS DEL AGUA SUBTERRÁNEA EN LA PARTE MEDIA DE LA CUENCA DEL RÍO TULIÁN, PUERTO CORTÉS, HONDURAS
}

\author{
HYDROCHEMICALAND ISOTOPIC PROPERTIES OF THE GROUNDWATER IN THE \\ MIDDLE PART OF THE TULIÁN RIVER WATERSHED, PUERTO CORTÉS, HONDURAS
}

\author{
*Tirza C. Contreras, Ingrid Vargas \& Elena Badilla \\ Universidad de Costa Rica \\ *Autora para contacto: tirza.contreras@gmail.com
}

(Recibido: 15/12/2011 ; aceptado: 11/06/2012)

\begin{abstract}
The present study identifies the hydrochemical and isotopic properties of 11 springs, including one thermal spring, in Tulian watershed, located in the city of Puerto Cortés, Cortés, Honduras. The relation between the concentrations of the environmental stable isotopes deuterium $\left({ }^{2} \mathrm{H}\right)$ and oxygen $-18\left({ }^{18} \mathrm{O}\right)$ in the water is similar to the relation in global meteoric water, indicating that the water has a meteoric origin. Evaluation of the geochemical characteristics of the water reveals that these springs belong to the same hydrogeological system, which comprises a fractured schist and gneiss of the Esquistos Cacaguapa formation. The water of the evaluated springs is mainly $\mathrm{Na}-\mathrm{Ca}-\mathrm{HCO}_{3}$. The $\delta^{2} \mathrm{H}(\%)$ and $\delta^{2} \mathrm{H}$ contents indicate that there is no variation in the \%o value.

Key words: hydrochemistry, oxygen -18 , deuterium, springs, thermal springs, Puerto Cortés.

RESUMEN: En este estudio se identifican las propiedades hidroquímicas e isotópicas del agua subterránea recolectada en 11 manantiales, 1 de ellos termal, en la parte media de la cuenca del río Tulián de la ciudad de Puerto Cortés, Cortés, Honduras. A partir de los valores de los isótopos estables, deuterio $\left({ }^{2} \mathrm{H}\right)$ y oxígeno $-18\left({ }^{18} \mathrm{O}\right)$, se deduce que el origen del agua subterránea es meteórico. Las características hidrogeoquímicas indican que los manantiales pertenecen a un mismo sistema hidrológico, compuesto por esquistos y gneiss fracturados de la formación Esquistos Cacaguapa. El agua de los manantiales evaluados es principalmente bicarbonatada sódica y bicarbonatada cálcica. Los contenidos isotópicos no muestran una variación de $\delta^{18} \mathrm{O}$ cómo se esperaría en el manantial termal.

Palabras clave: hidrogeoquímica, oxígeno-18, deuterio, manantiales, manantial termal, Puerto Cortés.
\end{abstract}




\section{INTRODUCCIÓN}

Tectónicamente, Honduras está situada en un margen activo (Bundschuh\& Alvarado, 2006) y particularmente, la zona de Puerto Cortés se localiza en la parte noroeste del bloque Chortis, al sureste de la zona de fallamiento Motagua Polochic; además, al noreste se ubica la falla del Chamelecón. Ambos sistemas de fallamiento hacen que la parte montañosa de esta cuidad Puerto, donde este estudio se concentra, sea dominado por fallas de orientación sudoeste - noreste, que corren subparalelas a los bandeamientos de esquistosidad de las rocas metamórficas que la conforman provocando el afloramiento de manantiales a través de estas fallas (Cálix, 2009). Se propone para la zona de estudio la presencia de la falla Agua Caliente ubicada en la parte media de la cuenca del río Tulián. En esta región, particularmente se da el afloramiento de manantiales termales. Estudios realizados en otras zonas del país describen los manantiales termales como manantiales que afloran a través de un sistema de convección hidrotermal, donde el agua meteórica se infiltra y es calentada en profundidad (Goff et al, 1987). Este estudio se concentra en la parte media de la cuenca del río Tulián (Fig. 1), e identifica las propiedades hidrogeoquímicas e isotópicas del agua subterránea en el área.

\section{MARCO GEOLÓGICO Y GEOMORFOLÓGICO}

La geología del área de estudio está compuesta básicamente por la formación conocida como Esquistos Cacaguapa, donde se incluye una gruesa secuencia de rocas metamórficas del Paleozoico que afloran en una amplia parte del Norte de Honduras (Servicio Nacional de Acueductos y Alcantarillados \& British Geological Service, 1994). La mayoría de los límites geológicos están controlados por fallas, mientras que las rocas consisten principalmente de filitas y esquistos micáceos de bajo grado, con cuarcita y mármol subordinados (Servicio Nacional de Acueductos y Alcantarillados \& British Geological Service,
1994). Además, aflora en el área un gneiss meteorizado, compuesto principalmente por cuarzo, feldespato y abundante muscovita y, hacia la parte baja de la cuenca, como producto de la meteorización y erosión de las rocas metamórficas, se observan materiales no consolidados de edad reciente, como arena y grava.

Los esquistos cubren un 66\% del área de estudio y se ubican a lo largo de casi toda el área de la cuenca, seguido por un $30 \%$ correspondiente a los gneiss ubicados en la parte media y un $4 \%$ de sedimentos cuaternarios en la parte baja de la cuenca. La geología regional del área de estudio se observa en la figura 2.

El análisis geomorfológico de la zona de estudio se realizó a partir del análisis de mapas topográficos y fotografías aéreas escala 1:40 000 obtenidos en el Instituto Geográfico Nacional. El área está compuesta básicamente por unidades de origen denudacional ubicadas hacia el centro y norte de la zona de estudio, representadas por laderas de suave a moderada pendiente, que presentan drenajes de tipo paralelo y rectangular debido al control estructural, evidenciado por la gran cantidad de lineamientos en dirección norte-sur y este-oeste que se ubican en la parte central del área de estudio. Hacia el norte y en la desembocadura del río Tulián al mar Caribe, se presenta una unidad de origen fluvial, un delta, que se compone de sedimentos producto de la denudación de los cuerpos montañosos de origen metamórfico.

Se propone la existencia de la Falla Agua Caliente en el área de estudio (Fig. 3), los argumentos que sustentan esta propuesta son los siguientes: la presencia de los lineamientos, el control estructural evidenciado en los ríos que desembocan al rio Agua caliente y que forman un ángulo de 90 grados respecto a este, la presencia de facetas triangulares en la parte media de la cuenca, la presencia de manantiales y fuentes termales alineadas este - oeste, el desplazamiento sinextral del río aguas abajo del sitio conocido como Los Encuentros y el valle lineal y estrecho del rio Tulián con rumbo este - oeste. Esta falla es oblicua al extremo noreste de la falla Motagua, por lo que se considera como una falla sintética a dicha falla y es de desplazamiento sinextral con 

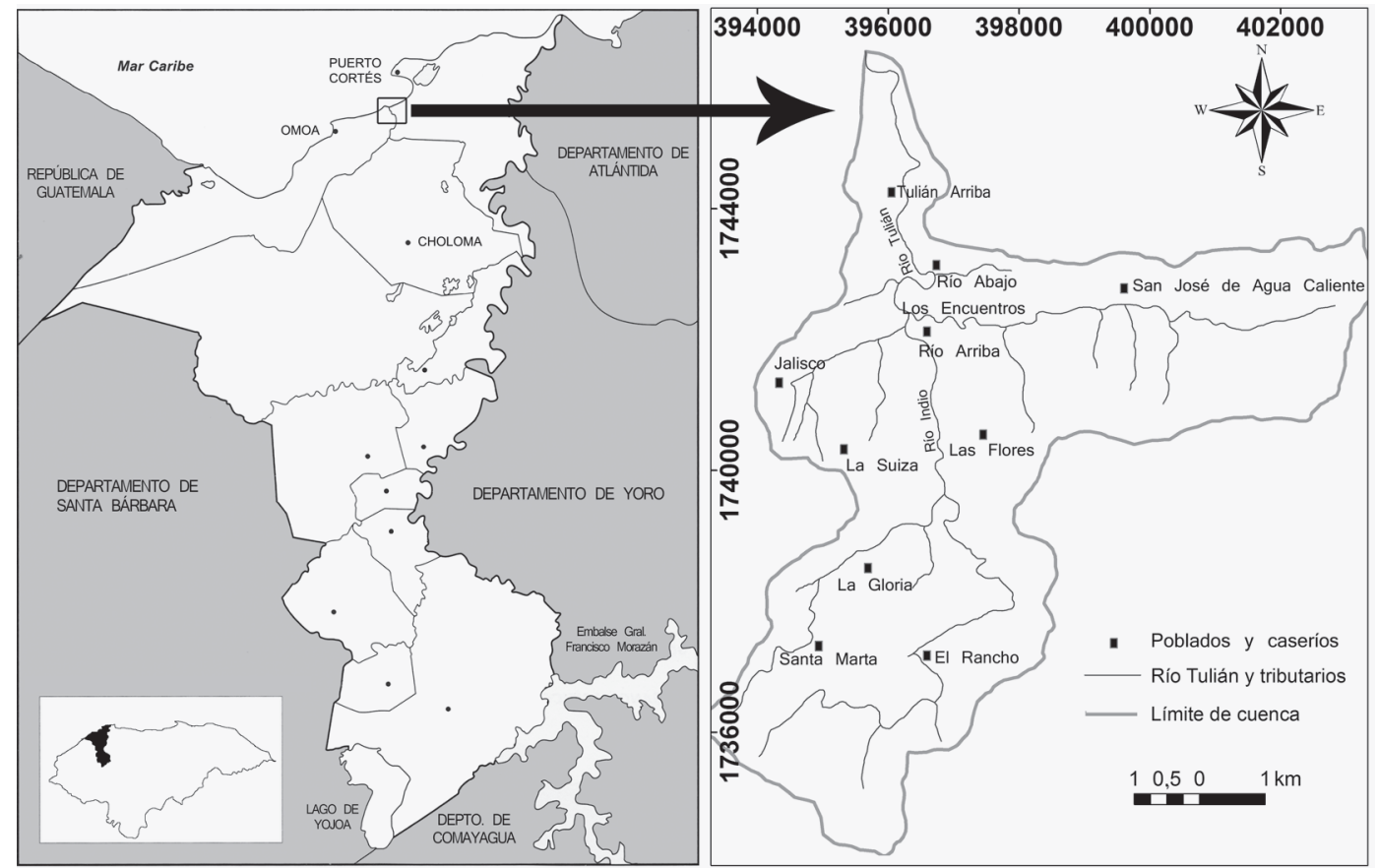

Fig. 1: Ubicación geográfica del área de estudio.

un componente vertical evidenciado por las facetas triangulares y diferencias de alturas del escarpe entre el margen izquierdo y derecho del cauce del rio Tulián.

\section{HIDROGEOLOGÍA}

Según Losilla et al. (2001), la utilización de agua subterránea en Honduras varía dependiendo de la zona; en general el uso de ésta fluctúa entre un $30 \%$ y un $100 \%$. En el caso de la ciudad de San Pedro Sula, la cual se localiza relativamente cerca del área de estudio, el $80 \%$ del agua utilizada es subterránea, con la máxima extracción promedio de agua subterránea en el país con 115200 m3/día. Esto se debe a que esta ciudad tiene una producción industrial alta debido a que en la ciudad es donde se da la principal actividad comercial del país. En contraste con San Pedro Sula, en las zonas rurales como es el caso de la zona de estudio, existen muy pocos pozos, las poblaciones en estas zonas se abastecen de manantiales y agua superficial.
El flujo regional del agua subterránea en la zona de estudio es hacia el norte, con un gradiente hidráulico de 0,16. En la parte media de la cuenca se dibujaron las líneas isofreáticas con base en la ubicación de manantiales, los cuales permanecen durante todo el año y rinden caudales promedio de $0,21 / \mathrm{s}$.

SANAA \& BGS (1994) definen para la zona de estudio la presencia de dos unidades hidrogeológicas, una unidad hidrogeológica que corresponde con la formación Esquistos Cacaguapa, la cual tiene una porosidad y permeabilidad primaria muy baja, y es la presencia de fracturas y del sistema de fallas que se presume existe en esta zona montañosa de Puerto Cortés, la que permite el afloramiento del agua subterránea, a través de los manantiales. La otra unidad hidrogeológica con acuíferos altamente productivos, corresponde al Aluvión del Valle de Sula y en la zona de estudio se ubica al norte, en la parte baja de la cuenca (SANAA \& BGS, 1994).

De acuerdo con las características geomorfológicas mencionadas, se identificó la falla Agua 
Caliente, en la comunidad de río Arriba; en esa zona también se localizan la mayor parte de los manantiales inventariados en el campo, algunos de los cuales fueron muestreados para realizar análisis hidrogeoquímicos e isotópicos. De esta forma se interpreta que esta es una zona de descarga del agua subterránea donde además hay presencia de aguas termales, como lo evidencian dos manantiales termales encontrados. La temperatura promedio de descarga de estos manantiales termales es de $60^{\circ} \mathrm{C}$, y tienen caudal aproximado de 0,021 1/s. Visitas realizadas al campo corroboran que las unidades litológicas están compuestas básicamente por esquistos y gneiss en la parte media de la cuenca.

\section{RESULTADOS DE ANALISIS HIDROGEOQUÍMICOS E ISOTÓPICOS}

\section{Metodología aplicada}

Con el objetivo de realizar una caracterización hidrogeoquímica de las aguas subterráneas del sistema acuífero en estudio, se realizó una campaña de toma de muestras de agua para hacer análisis químicos e isotópicos en junio del año 2009, en total se recolectaron y analizaron 11 muestras de agua, todas provenientes de manantiales (Figura 4). La mayoría de las muestras se tomaron en la parte media de la cuenca, excepto por dos muestras que se tomaron en la parte alta de la cuenca, con el fin de tener una firma isotópica a una altitud diferente y conocer además si las propiedades hidrogeoquímicas eran similares en el área de estudio. Los análisis químicos que se realizaron incluyen aniones $\left(\mathrm{HCO}^{3-}, \mathrm{SO}^{4-}\right.$ y Cl$\left.{ }^{-}\right)$ y cationes mayoritarios $\left(\mathrm{Ca}^{2+}, \mathrm{Mg}^{2+}, \mathrm{Na}^{+}, \mathrm{K}^{+}\right)$. Se utilizó para todos los casos métodos que están listados en el Estándar Métodos de la 20 edición que para los parámetros analizados utiliza métodos de volumetría para el $\mathrm{HCO}^{3-}$, el $\mathrm{Mg}^{2+}, \mathrm{Cl}^{-} \mathrm{y}$ $\mathrm{Ca}^{2+}$; métodos de colorimetría para el $\mathrm{SO}^{-}, \mathrm{Na}^{+}$y $\mathrm{K}^{+}$. En el campo se midieron los siguientes parámetros: $\mathrm{pH}$, conductividad eléctrica y temperatura, utilizando el equipo multifuncional facilitado por la Escuela Centroamericana de Geología

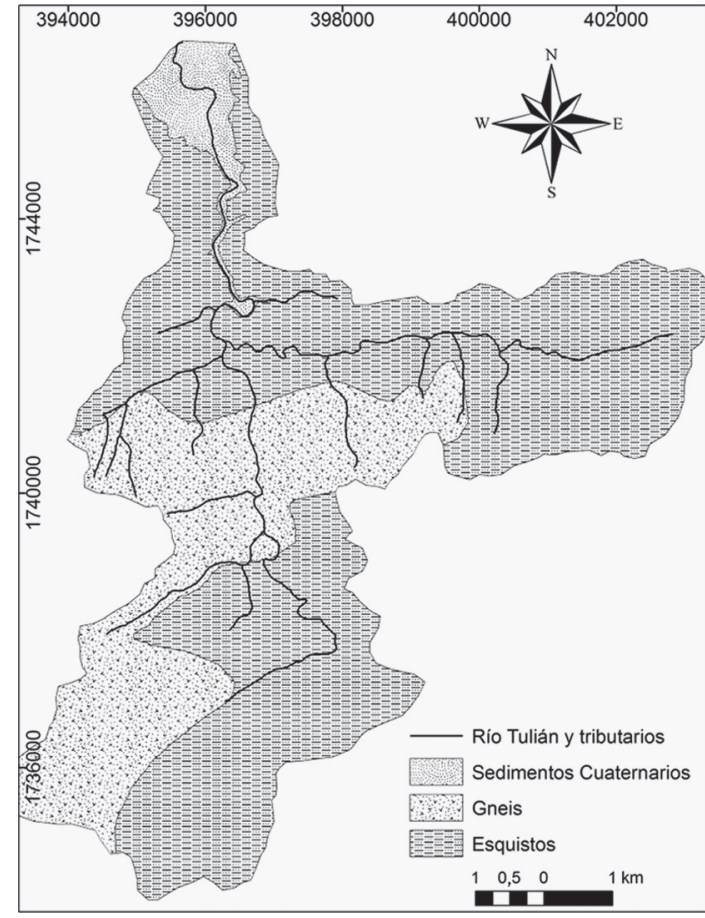

Fig. 2: Geología de la cuenca del río Tulián.

de la Universidad de Costa Rica, a través del Laboratorio de Geoquímica. Posteriormente, se recolectaron las muestras de agua en recipientes de plástico de 1 galón, los cuales fueron facilitados por el Laboratorio de Aguas de San Pedro Sula para realizar los análisis químicos indicados; se cumplió con el protocolo de muestreo establecido por dicho laboratorio. Se tomó además un duplicado de la muestra M7J que corresponde a la muestra M8J, para verificar la calidad del análisis en laboratorio y como se verá en los resultados, el resultado fue el esperado. Simultáneamente, se tomaron muestras para realizar los análisis isotópicos, de oxígeno $18\left({ }^{18} \mathrm{O}\right)$ y deuterio $\left({ }^{2} \mathrm{H}\right)$. Las muestras se tomaron considerando todas las indicaciones del laboratorio La Geo de El Salvador, el cual está certificado para realizar este tipo de análisis.

Este es el primer estudio que reporta datos hidrogeoquímicos e isotópicos del agua subterránea en esta zona, por lo que para el análisis isotópico se trabajó con la línea meteórica global propuesta por Clark \& Fritz (1997). 


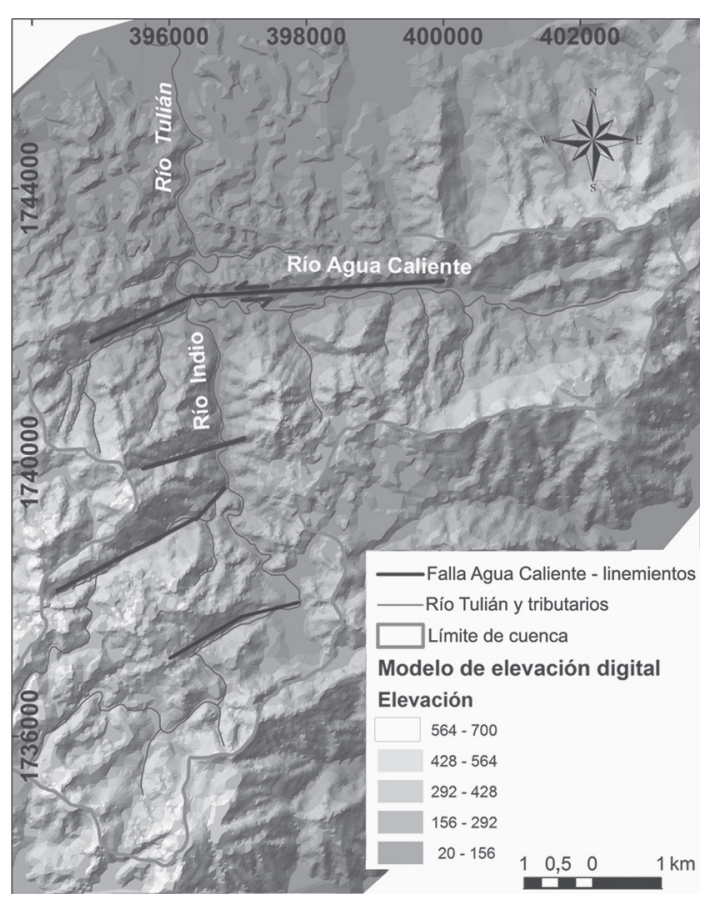

Fig. 3: Trazo de Falla Agua Caliente.

\section{Evaluación hidrogeoquímica}

En general, los parámetros físico - químicos de las muestras indican una similitud en todas ellas, indicando la naturaleza alcalina del agua presente en la región; de igual forma, las conductividades eléctricas y temperatura en la región son muy similares entre sí, a excepción en todos los casos de la muestra M5RA (manantial termal), tal como se muestra en el cuadro 1.

Las concentraciones iónicas de las aguas en el área de estudio son muy similares entre sí (cuadro 2). El rasgo más llamativo es la muestra M5RA, ya que, químicamente hablando es diferente a las demás, con una diferencia de temperatura respecto a las demás muestras de unos $40^{\circ} \mathrm{C}$.

La conductividad eléctrica de los 11 manantiales evaluados varía entre 86 y $300 \mu \mathrm{s} / \mathrm{cm}$, a excepción de la muestra M5RA, la cual tiene un valor de $1800 \mu \mathrm{s} / \mathrm{cm}$ y corresponde con un manantial termal. El orden de abundancia de aniones en el agua de los manantiales muestreados es: $\mathrm{HCO}^{3-}>\mathrm{Cl}^{-}>\mathrm{SO}_{4}=$, y los cationes más abundantes son el $\mathrm{Na}^{+}{\text {y } \mathrm{Ca}^{2+} \text { seguido del } \mathrm{Mg}^{2+} \text { y el K}}^{+}$. De acuerdo con el diagrama de Piper mostrado en las Figura 5, las aguas de los manantiales provienen de una fuente similar, y se clasifican principalmente como bicarbonatadas sódicas y bicarbonatadas cálcicas, por lo que se puede decir (dada la predominancia del bicarbonato) que, de acuerdo a la secuencia de Chevotareb (Freeze \& Cherry, 1979), estas aguas tienen un corto tiempo de permanencia en el terreno. La presencia de sodio se puede correlacionar con dos factores importantes. En primer lugar, no se descarta que estas concentraciones sean el resultado de la influencia del aerosol de agua marina; sin embargo, hay que tener en cuenta que las aguas circulan por un medio geológico en donde existen granitos metamorfizados (gneiss) y según los resultados de los análisis petrográficos, los gneiss tienen plagioclasas, y ortosa en su mayoría, minerales que también puede contribuir a las altas concentraciones de sodio. Asimismo, tal como lo afirma Custodio \& Llamas (2001), el resultado de algunas relaciones

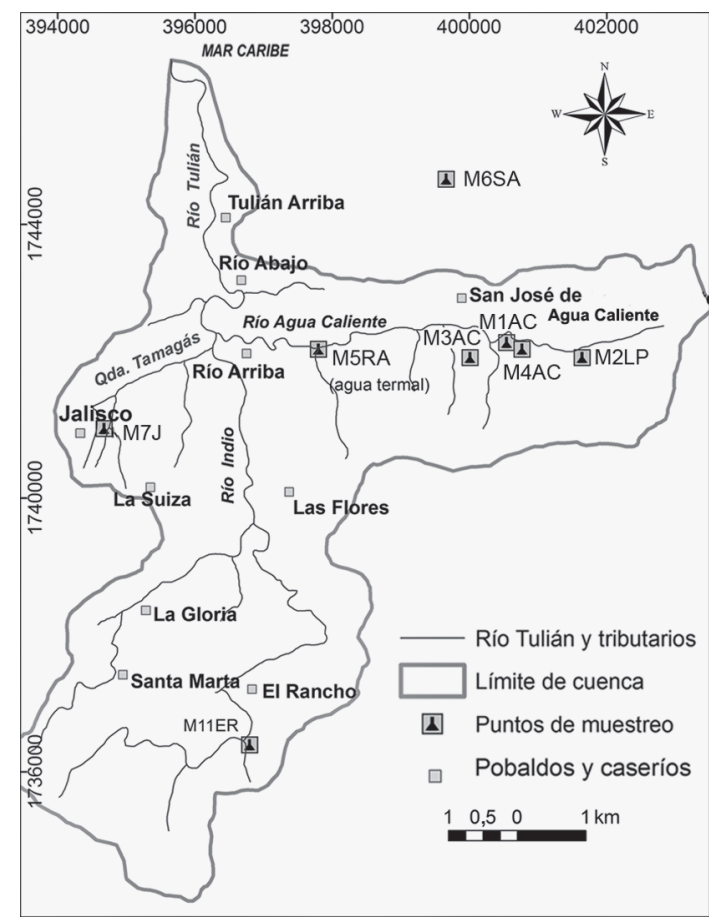

Fig. 4: Ubicación de muestras tomadas. 
Cuadro 1

Descripción y resultados de parámetros tomados en campo de los puntos de muestreo

\begin{tabular}{|c|c|c|c|c|c|c|c|c|c|c|c|}
\hline Muest. & Fecha & Tipo & Cód. & Ubicación & $\begin{array}{l}\text { Longi- } \\
\text { tud }\end{array}$ & Latitud & $\begin{array}{l}\text { Altitud } \\
(\mathrm{msnm})\end{array}$ & $\mathrm{pH}$ & $\begin{array}{l}\text { Temp. } \\
\left({ }^{\circ} \mathrm{C}\right)\end{array}$ & $\begin{array}{l}\text { Conduct. } \\
(\mathrm{Ms} / \mathrm{cm})\end{array}$ & $\mathrm{icb}$ \\
\hline 1 & $11 / 06 / 09$ & Mntl & M1AC & $\begin{array}{c}\text { Agua } \\
\text { Caliente }\end{array}$ & 400547 & 1742275 & 134 & 7,1 & 26 & 170 & $-5,97$ \\
\hline 2 & $11 / 06 / 09$ & Mntl & M2LP & Las Pilas & 401647 & 1742055 & 173 & 7,5 & & 300 & $-5,03$ \\
\hline 3 & $11 / 06 / 09$ & Mntl & M3AC & $\begin{array}{c}\text { Agua } \\
\text { Caliente }\end{array}$ & 400010 & 1742055 & 112 & 7,2 & 24 & 250 & $-4,52$ \\
\hline 4 & $11 / 06 / 09$ & Mntl & M4AC & $\begin{array}{c}\text { Agua } \\
\text { Caliente }\end{array}$ & 400771 & 1742176 & 104 & & & & $-7,33$ \\
\hline 5 & $11 / 06 / 09$ & Mntl & M5RA & $\begin{array}{l}\text { Río A (agua } \\
\text { termal) }\end{array}$ & 397800 & 17422176 & 80 & 5,9 & 60 & 1800 & $-22,17$ \\
\hline 6 & $12 / 06 / 09$ & Mntl & M6LM & La Mota & & & & 8,32 & 24,3 & & $-3,48$ \\
\hline 7 & $12 / 06 / 09$ & Mntl & M7J & Jalisco & 394678 & 1741017 & 259 & 7,9 & 24,3 & 250 & $-1,76$ \\
\hline 8 & $12 / 06 / 09$ & Mntl & M8J & Jalisco & 394678 & 1741017 & 259 & 7,9 & 24,3 & & $-1,71$ \\
\hline 9 & $12 / 06 / 09$ & Mntl & M9SA & San Antonio & 399664 & 1744662 & 141 & 7,5 & 25,6 & 116 & $-3,22$ \\
\hline 10 & $13 / 06 / 09$ & Mntl & M10ER & El Rancho & & & 413 & 7,8 & 24,4 & 86 & $-2,08$ \\
\hline 11 & $13 / 06 / 09$ & Mntl & M11ER & El Rancho & 396798 & 1736393 & & 7,65 & 24,1 & 96 & $-1,38$ \\
\hline
\end{tabular}

Muest: muestra; mntl: manatial; cód: código; Temp: temperatura; conduct: conductividad

iónicas ayuda a visualizar los efectos que se pueden estar produciendo en el terreno. De éstas, una de las más importantes es el índice de cambio de bases (icb), cuyos resultados se muestran en el cuadro 1; un aumento o disminución de esta relación indica un cambio de bases del agua: si el índice disminuye indica un cambio en las bases de ablandamiento y si ese índice aumenta el cambio, representa un endurecimiento del agua. Los resultados de las muestras que se tomaron, indican valores claramente negativos, lo cual puede ser indicador de un intercambio catiónico, fenómeno especialmente asociado con la presencia de minerales de arcilla, que afecta principalmente los cationes $\mathrm{Na}^{+}, \mathrm{Ca}^{+}, \mathrm{y} \mathrm{Mg}^{2}+$, y que consiste en que el terreno cede iones $\mathrm{Na}+\mathrm{y}$ toma del agua iones $\mathrm{Ca}^{+}, \mathrm{y} \mathrm{Mg}^{2+}$; como resultado, el agua se ablanda y tiende a convertirse en sódica.

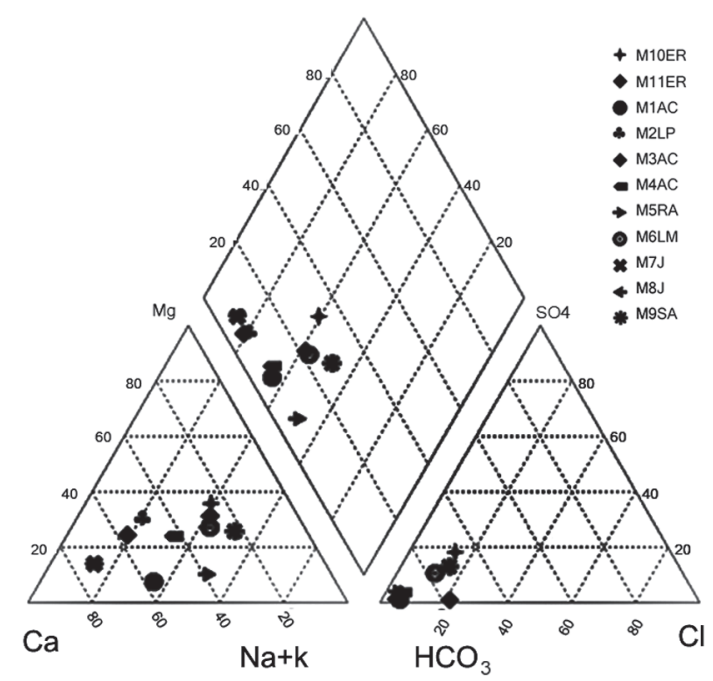

Fig. 5: Diagrama de Piper. 
Cuadro 2

Resultados análisis químicos

\begin{tabular}{cccccccccc}
\hline \# muestra & Fecha & muestra & $\begin{array}{c}\mathrm{Na} \\
(\mathrm{mg} / \mathrm{L})\end{array}$ & $\begin{array}{c}\mathrm{K} \\
(\mathrm{mg} / \mathrm{L})\end{array}$ & $\begin{array}{c}\mathrm{Mg} \\
(\mathrm{mg} / \mathrm{L})\end{array}$ & $\begin{array}{c}\mathrm{Ca} \\
(\mathrm{mg} / \mathrm{L})\end{array}$ & $\begin{array}{c}\mathrm{Cl} \\
(\mathrm{mg} / \mathrm{L})\end{array}$ & $\begin{array}{c}\mathrm{SO}_{4} \\
(\mathrm{mg} / \mathrm{L})\end{array}$ & $\begin{array}{c}\mathrm{HCO}_{3} \\
(\mathrm{mg} / \mathrm{L})\end{array}$ \\
\hline 1 & $11 / 06 / 09$ & M1AC & 20,9335 & 2,8153 & 2,38 & 31,68 & 5 & 0,88 & 135 \\
2 & $11 / 06 / 09$ & M2LP & 15,2325 & 2,6067 & 12,92 & 34,85 & 5 & 4,674 & 171 \\
3 & $11 / 06 / 09$ & M3AC & 10,9127 & 1,9012 & 8,08 & 31,68 & 4 & 1,108 & 140 \\
4 & $11 / 06 / 09$ & M4AC & 22,1649 & 3,5063 & 9,03 & 26,93 & 5 & 4,685 & 156 \\
5 & $11 / 06 / 09$ & M5RA & 190,1476 & 34,3119 & 21,86 & 139,39 & 14 & 22,59 & 676 \\
6 & $12 / 06 / 09$ & M6LM & 13,0526 & 2,5212 & 4,75 & 8,71 & 5 & 5,794 & 55 \\
7 & $12 / 06 / 09$ & M7J & 8,0966 & 1,4318 & 4,75 & 41,18 & 5 & 1,596 & 150 \\
8 & $12 / 06 / 09$ & M8J & 7,9367 & 1,4232 & 4,75 & 41,18 & 5 & 1,253 & 140 \\
9 & $12 / 06 / 09$ & M9SA & 15,3313 & 1,8497 & 4,28 & 6,34 & 6 & 6,994 & 50 \\
10 & $13 / 06 / 09$ & M10ER & 9,157 & 1,4312 & 4,75 & 5,54 & 5 & 8,385 & 40 \\
11 & $13 / 06 / 09$ & M11ER & 9,8381 & 1,6413 & 4,28 & 6,34 & 7 & 0,287 & 44 \\
\hline
\end{tabular}

Esta es una hipótesis para esta área de estudio, ya que esta evaluación deberá hacerse a lo largo del flujo en el área de la cuenca.

\section{Composición Isotópica}

Las composiciones isotópicas de deuterio $\left({ }^{2} \mathrm{H}\right)$ y oxígeno $-18\left({ }^{18} \mathrm{O}\right)$ para la zona de estudio se aproximan a los valores isotópicos presentadas en la línea meteórica global. Los resultados para el oxígeno- 18 varían de $-3,7 \%$ a $-4,2 \%$ y para el deuterio se presenta un rango más amplio de $-12,9 \%$ a $-19 \%$ y se estima un error de $\pm 1 \%$ o para el deuterio y de $\pm 0,2 \%$ para el oxígeno- 18 . El hecho de que los valores isotópicos se localicen cerca de la línea meteórica global, indica que las aguas de los manantiales en la parte media de la cuenca son de origen meteórico. El valor de $\delta^{18} \mathrm{O}$ para la precipitación en esta zona de Honduras, de acuerdo a la red de estaciones globales que monitorean las precipitaciones, instaladas por el Organismo Internacional de Energía

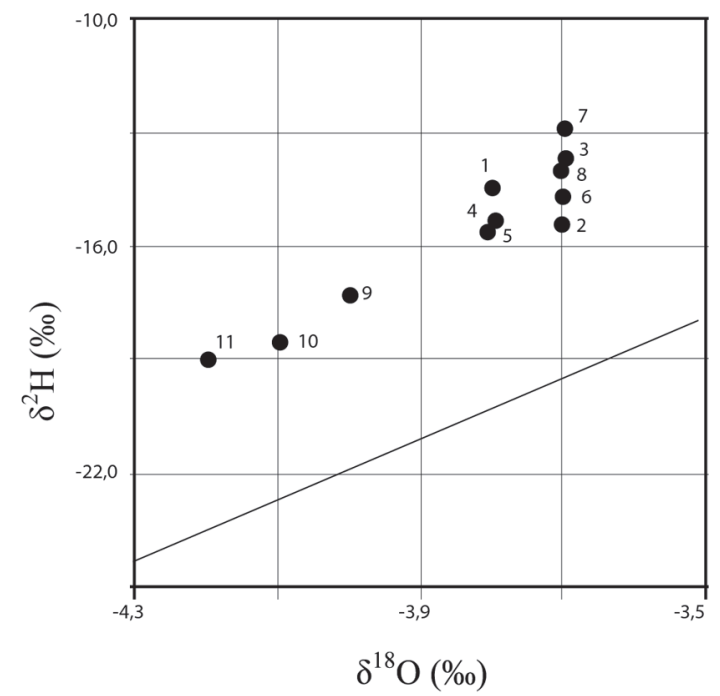

Fig. 6: Resultados Isotópicos. Los números de muestra corresponden con los cuadros.

Atómica (OIEA), es del orden de $-6 \%$ (L. Toro, com. esc., 2010). De acuerdo con este valor, se puede determinar que las aguas están enriquecidas 


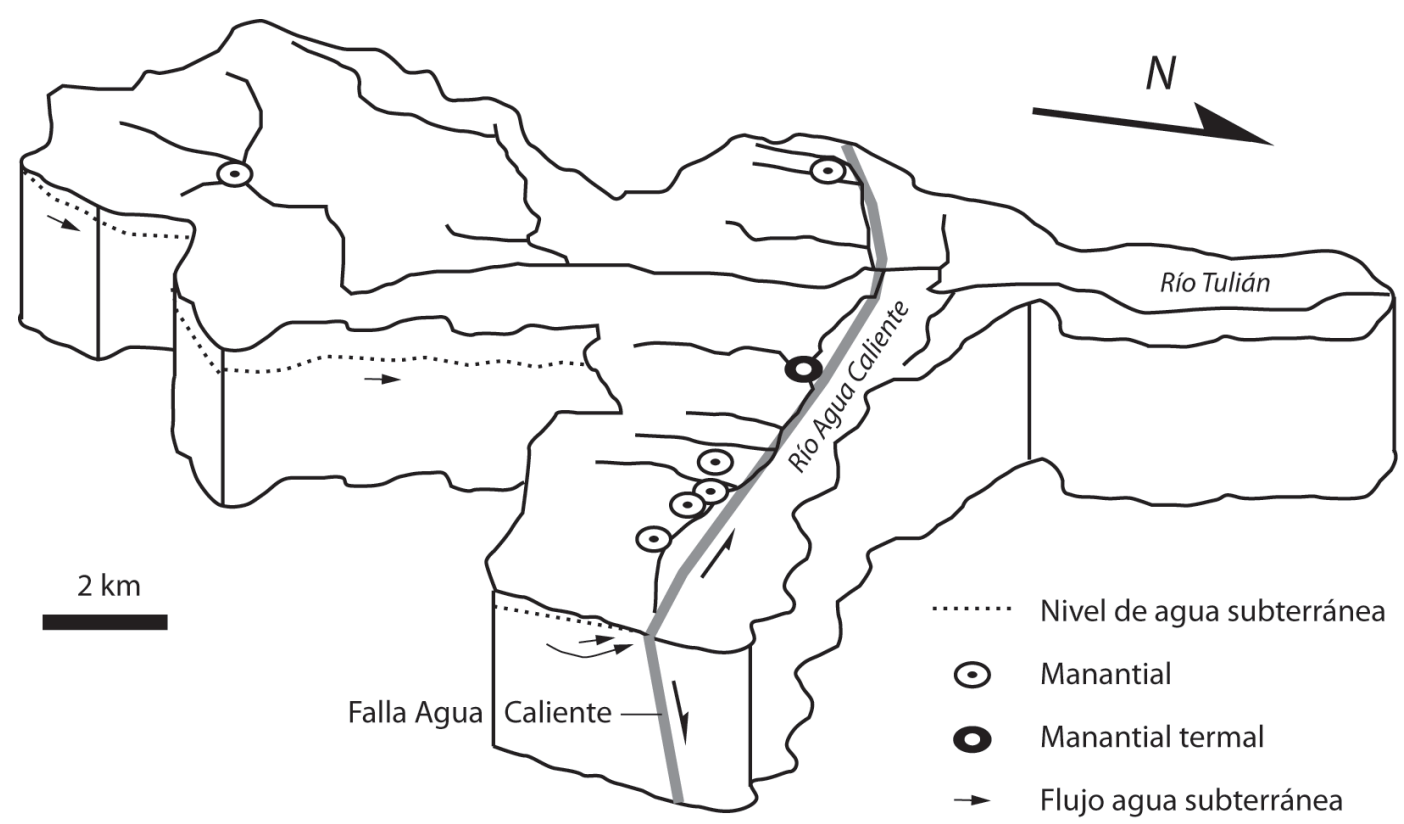

Fig. 7: Modelo conceptual.

en ${ }^{18} \mathrm{O}$ debido posiblemente a un proceso de evaporación propio del ciclo hidrológico. Como se observa, las muestras M1AC a M7J corresponden prácticamente al mismo tipo de agua, lo cual es esperado debido a la cercanía entre los puntos de muestreo. Por otro lado, se observa también en la figura 6 que la muestra M5RA no se diferencia del resto de las muestras, cómo se suponía que sucedería debido a que esta sería agua termal y sus características químicas respaldan esta hipótesis; sin embargo, el resultado isotópico esperado sería un enriquecimiento importante del oxígeno 18 y no es así, por lo que se puede concluir entonces que el agua que aflora en este punto es agua de lluvia que se ha infiltrado y pasa a través de una falla donde se calienta, y aflora a las superficie con propiedades químicas diferentes a las demás muestras. El modelo conceptual propuesto en la Figura 7 ilustra este proceso. Las muestras M9SA y M10ER parecen corresponder a otro grupo de composición isotópica, aunque realmente su diferencia no es muy notable con relación al primer grupo (muestras M1AC-M7J); la muestra M10ER corresponde al manantial con mayor altitud y su firma isotópica ligeramente empobrecida respecto a las otras muestras, podría ser fruto del factor altitud sobre las aguas lluvias que se han infiltrado y ahora descargan a través de este manantial. La muestra M9SA fue tomada fuera del área de la cuenca con el objeto de tener un punto cercano al área y conocer si las propiedades tanto químicas como isotópicas son similares en las comunidades aledañas, sin embargo, para este efecto se deben tomar en cuenta puntos más dispersos a lo largo del área. Todas las muestras están aproximadamente alineadas y podrían corresponder al mismo tren de humedad que genera la precipitación en la cuenca. 


\section{CONCLUSIONES}

En general, la composición química de las aguas de los manantiales muestreados particularmente a lo largo del río Agua Caliente son bicarbonatadas sódicas y cálcicas, lo cual es muy típico de zonas de recarga. Y de acuerdo con la serie de Chevotareb corresponden a aguas que tienen un corto tiempo de permanencia en el terreno.

De acuerdo con los resultados del cálculo del índice de bases se presume que a lo largo del flujo se está dando un intercambio catiónico que podría ser el responsable de que las aguas muestreadas en algunos puntos sean bicarbonatadas sódicas.

A excepción de una muestra, todas tienen concentraciones de iones mayores promedio. La muestra M5RA muestra una composición química muy diferente a las demás, incluso su temperatura supera en casi $40^{\circ} \mathrm{C}$ a las demás muestras. Se pensó que era agua termal que emergía de profundidad, sin embargo esta hipótesis es refutada por la firma isotópica de la muestra, ya que si así fuera se hubiese observado un enriquecimiento importante del $\mathrm{O}^{18}$ y no es así. La elevada temperatura probablemente se deba a que antes de su descarga el agua pasa por un proceso de calentamiento que se presume se da en el momento que el agua asciende a través de la falla inferida a lo largo del cauce del rio Agua Caliente, lo cual corresponde con un comportamiento propio de un sistema de convección hidrotermal.

Los análisis isotópicos demuestran que todas las muestras corresponden al mismo tren de humedad que genera la precipitación en la zona de estudio, lo que sugiere que las aguas son de origen meteórico.

\section{AGRADECIMIENTOS}

Al DAAD por el financiamiento brindado, a Ronald Ramírez y Luis Obando por el apoyo con las descripciones petrográficas de las muestras de roca. Los comentarios de Percy Denyer y Walter
Montero fueron muy valiosos. A Luis Toro, del OIEA, por su valiosa colaboración en la interpretación isotópica de las muestras analizadas. A los pobladores de las diferentes comunidades visitadas por su apoyo, a la Empresa Aguas de Puerto Cortés y a la Municipalidad de Puerto Cortés por el apoyo logístico brindado.

\section{REFERENCIAS BIBLIOGRÁFICAS}

BUNDSCHUH, J., ALVARADO G.E., 2007: Central America Geology, Resources, Hazards, Volume 1. - 647 págs. Taylor \& Francis, London.

CALIX, R., 2009: Estudio Geológico, geotécnico y de la incidencia sísmica de la península de Puerto Cortés.- 60 págs. Municipalidad de Puerto Cortés, Honduras. [Inf. Interno]

CLARK, I., \& FRITZ, P., 1997: Environmental Isotopes in Hydrogeology. - 328 págs. Lewis Publishers, New York.

CUSTODIO, E., \& LLAMAS, M.R., 2001: Hidrología Subterránea (2 ${ }^{\mathrm{a}}$ ed.).- 1157 págs. Ediciones Omega, Barcelona.

FREEZE, A. \& CHERRY, J.A., 1979: Groundwater. - 604 págs. Prentice Hall, New Jersey.

GOFF, F.E., TRUESDELL, AH., GRIGSBY, C.O., JANIK, C.J., SHEVENELL, L.A., PAREDES, J.R., GUTIERREZ, J.W., PE, JR, COUNCE, D.A., 1987: Hydrogeochemical investigation of six geothermal sites in Honduras, Central America.- 170 págs. Los Alamos National Laboratory, New Mexico.

LOSILLA, M., RODRÍGUEZ,H.,SCHOSINSKY, G., STIMSON, J., BETHUNE, D., 2001: 
Los acuíferos volcánicos y el desarrollo sostenible en América Central.- 205 págs. Universidad de Costa Rica, San José.
SANAA \& BGS, 1994: Mapa Hidrogeológico de la Zona Norte de Honduras.- Escala 1: 250,000, Instituto Geográfico Nacional, Tegucigalpa. 